\title{
The Loopholes in Chinese Law Reflected in the First Genetically Edited Babies
}

\author{
Ni Zhang* and Xueke Wang \\ China Jiliang University, China \\ *Corresponding author: Ni Zhang, Associate Professor of Law, China Jiliang University, China
}

To Cite This Article: Ni Zhang, Xueke Wang. The Loopholes in Chinese Law Reflected in the First Genetically Edited Babies. Am J Biomed Sci \& Res. 2019 - 1(6). AJBSR.MS.ID.000552. DOI: 10.34297/AJBSR.2019.01.000552

Received: March 01, 2019 | Published: March 13, 2019

\section{Opinion}

He Jiankui, an associate professor at the Southern University of Science and Technology of China was named one of the top ten technology persons by the British Science Journal "Nature" in its electronic version in 2018, and he was selected because of the genetically edited babies that had received widespread condemnation both in China and around the world. The "Science" magazine even ranked this incident as the first of three scientific breakdowns in 2018.

\section{Whole story of genetically edited babies of He Jiankui}

On November 26, 2018, the team of He Jiankui announced that two genetically edited babies conducted by their team were born in China. On November 28, He Jiankui claimed at the 2nd International Summit of Human Genome Editing that two little girls who had been genetically edited, "Lulu and Nana were born healthy." He admitted that the experimental data was leaked, and the research was not peer-reviewed. His team conducted several human trials ( 7 couples, 31 eggs, 70\% embryos), one of which realized knockout of CCR5, two gene sequences were improved as expected and Lulu and Nana were born. Although gene sequencing found a potential off-target risk, it was far from other genes and the parents of the babies were informed. He stated that the experiment was conducted to satisfy the needs of HIV parents who want healthy babies.

The event has undoubtedly detonated China and the global scientific community. How could they conduct such an experiment that may pose a huge potential risk to humans? Was it permitted by the related departments? Did He Jiankui involve with certain economic benefit? Too many questions left unexplained.

Without any ethical review, it is reported that He Jiankui's research started in March 2017, he contacted an AIDS community organization in Beijing to recruit volunteers for his experiment, and over 200 couples signed up for the project. After strict inspiring, his team obtained more than 50 couples' information satisfying the requirements, with the permission of clients 7 couples with HIV father-positive and mother-negative were selected by the team, and He Jiankui started the experiment and published the results for the first time in more than a year, claiming that another subject was pregnant.

\section{Questions about the event}

Is genome-editing technique a mature and significant disease treatment technology, is it permitted by Chinese law?

He Jiankui claimed that his team used the CRISPR/Cas9 to modify the CCR5, and there are about $10 \%$ Northmen have a natural CCR5 gene deletion. People with such mutation can close the gate for the most pathogenic HIV infection, so that the virus can't invade human cells, in other words, they can naturally immunize HIV. Simply knocking out the gene may reduce certain risk of AIDS; however, such genetic editing cannot fully mimic the natural loss of the CCR5, and it can't exclude the possibility of causing other diseases.

Genetic editing is a new technology in China. In February 2001, the Ministry of Health of China promulgated the "Management Measures for Human Assisted Reproductive Technology" and the "Management Methods for Human Sperm Banks" and released the "Human Assisted Reproductive Technical Specifications", "Human Sperm Library Basics" "Human Sperm Library Technical Specification" and the "Ethical Principles for the Implementation of Human Assisted Reproductive Technology" in May 2001. After that, with the development of biotechnology and the renewal of social ethics, in 2003, the Ministry of Health formed the "Ethical Principles of Human Assisted Reproductive Technology and Human Sperm Bank" on the basis of the revision of the "Ethical Principles for the Implementation of Human Assisted Reproductive Technology", which further refined the ethical principles in application of the technology, and also revised the "Human Assisted Reproductive Technical Specifications" and "Basic Standards and Technical 
Specifications for Human Sperm Banks", the original "Human Assisted Reproductive Technology Management Measures" and "Ethical Principles for the Implementation of Human Assisted Reproductive Technology" were abolished simultaneously. According to the revised regulations, human assisted reproductive technologies in China as mainly referred to as in vitro fertilizationembryo transfer and its derivative technologies, including in vitro fertilization-embryo transfer, gamete or zygote transplantation, intracytoplasmic sperm injection, embryo cryopreservation, genetic diagnosis of fusion and preimplantation embryos, and genetic editing is not included. Therefore, it is concluded that there is no legal basis for gene editing in China, but the experiments conducted by The Jiankui's team involve in vitro fertilization and embryo transfer, etc., and it should abide by the regulations.

\section{Is the experiment an inevitable choice and does it require an} ethical review?

According to the views of insiders, the genetically edited babies are not necessary; based on current medical level, for a couple with HIV father-positive and mother-negative, the washing + blocking technology can avoid nearly $100 \%$ of children's genetic AIDS. In addition, professionals both at home and abroad also disclosed that the genetic editing of the experiment was a failure. Nana, one of the twins, was actually off-target and Nana actually became the control group. Knowingly there were many mistakes, and He Jiankui still gave birth to the babies.

Does such a risky experiment need an ethical review? The "Ethics Review Method for Human Biomedical Research" (hereinafter referred to as "the "Measures for Review") published by National Health and Family Planning Commission in 2016 clearly states: "Medical and health institutions engaged in biomedical research involving human beings is the management main body for ethical review, an ethics committee should be established and effective measures should be taken to ensure that the committee can carry out its work independently." "Administrative Measures for Clinical Research Projects of Medical and Health Institutions" (December 2013) stipulates that clinical research shall be applied by medical personnel who are legally practicing at the medical and health institutions, and 12 materials such as the application for the project and the qualification certificate of the applicant are required, besides, the medical and health institution should establish an ethics committee and form a written review record and opinions. Therefore, the experiments conducted by He Jiankui must go through a prior ethical review.

A registration document entitled "HIV Immunization Gene CCR5 Embryo Gene Editing Safety and Validity Evaluation" registered in the China Clinical Trial Registration Center suggested that the registration time was November 2017, the applicant was South University of Science and Technology of China, one of the contacts is He Jiankui, the implementation site of the research is Harmonicare women \& children's hospital, and the ethics committee is Shenzhen Harmonicare women \& children's hospital, in the attachment there is a "Application for Examination of the Medical Ethics committee of Shenzhen Harmonicare women \& children's hospital". About the incident on November 26, 2018, however, the Southern University of Science and Technology stated that He Jiankui had been suspended from work on February 1, 2018, and the university had no knowledge of the research; Shenzhen Harmonicare women \& children's hospital denied the ethics committee had approved the application on March 7 and said that the experiment was never conducted, the hospital claimed that someone forged the signature and they had handled it to the police. Shenzhen Science and Technology Innovation Committee has also denied that the research funding or other resources were provided by "Shenzhen Science and Technology Innovation Free Exploration Project", mentioned in the "registration document". Therefore, He Jiankui's experiment has not been approved by the relevant departments, and the application documents are likely to be forged.

Meanwhile, the incident also indicated that there are many loopholes in the ethical review of China: such as what are the entry criteria for members of the ethics committees of medical institutions, which department is responsible for the supervision of the hospital's ethics committee, and what legal responsibility to bear if the ethics committee fail to fulfill its responsibility, etc.

\section{What legal responsibility does He Jiankui have to bear?}

Since the above-mentioned laws and regulations related to human medicine and reproductive technology are formulated by the Chinese health and medical authorities, the main subjects involved are medical personnel including doctors, nurses and related personnel, and He Jiankui as a researcher, whether he has to bear corresponding criminal responsibility is still a question. 